\title{
ESTIMACIÓN DE LA COMPETITIVIDAD DE LOS PAÍSES EXPORTADORES DE FRUTA DEL HEMISFERIO SUR A PARTIR DEL ÍNDICE DE COMPETITIVIDAD PARA EL CRECIMIENTO EN EL PERÍODO 2001-2004
}

\author{
A COMPETITIVENESS EVALUATION OF FRUIT FROM THE EXPORTING \\ COUNTRIES OF THE SOUTHERN HEMISPHERE, ACCORDING TO THE \\ GROWTH COMPETITIVENESS INDEX IN THE PERIOD 2001-2004
}

\author{
Luis Tapia Iturrieta ${ }^{1}$
}

\begin{abstract}
RESUMEN
Este estudio tiene como objetivo analizar la competitividad generada por los países exportadores de fruta del hemisferio sur a partir de la información que entrega el índice de competitividad para el crecimiento, que calcula el Foro Económico Mundial, y correlacionar esta información con la competencia en la participación de mercado de las cinco especies más importantes de las exportaciones frutícolas chilenas (XF5). Este tipo de análisis permitió establecer cuadrantes característicos donde se encuentran los países que lideran las producciones, estableciéndose una ruta crítica para la promoción de liderazgo propio del país y para cada especie.

Palabras clave: Competitividad, índice de competitividad para el crecimiento, productores de fruta del hemisferio sur, liderazgo por especies: uva, manzanas, peras, kiwi y paltas.
\end{abstract}

\section{SUMMARY}

The main objective of this paper was to analyze the competitiveness generated by the fruit countries of the southern hemisphere, according to the information gives by the index of competitiveness fors the growth. This information is calculated by the World Economic Forum and is correlated with the competence of the five most important Chilean fruit exports (XF5) in market participation. This type of analysis allowed us to establish characteristic quadrants, where the countries that lead the productions were revealed, creating a critical route for the promotion of the country's own leadership for each fruit species.

Key words: Competitiveness, Index of competitiveness for the growth, producers of fruit of the South hemisphere, leadership by species: grape, apples, pears, kiwi and avocado.

\section{INTRODUCCIÓN}

La competitividad de las naciones se ha podido expresar en un conjunto de variables e indicadores como los que aplica el Foro Económico Mundial, que calcula el Índice de Competitividad Global que emplea dos enfoques complementarios para el análisis de la competitividad. Así, el Î́ndice de Competitividad para el Crecimiento (ICC) mide la capacidad de una economía nacional para lograr un crecimiento económico sostenido en el mediano plazo, en función del ambiente macroeconómico, las instituciones públicas y la tecnología y, por otro lado, el Índice de Competitividad para los Negocios (ICN) que evalúa la eficiencia con que una economía utiliza un stock de recursos en función de las operaciones y estrategias de empresas, además del ambiente nacional de negocios. Estos índices permiten efectuar un ranking de las naciones para mostrar un espectro de la diversidad competitiva de ellas (Foro Económico Mundial, 2004).

Por su parte, el profesor M. Porter (1991) desarrolla un modelo que permite explicar por qué las naciones son capaces de generar y mantener

1 Facultad de Ciencias Agronómicas, Universidad de Tarapacá, 1tapia@ uta.cl

Fecha de Recepción: 02 Mayo 2006

Fecha de Aceptación: 09 Junio 2006 
sus posiciones competitivas durante considerables periodos de tiempo. Además, explica cómo puede influir el entorno (políticas económicas del país, sus instituciones) en el éxito competitivo de sus empresas y diferenciar cuáles son los factores que fomentan las ventajas competitivas del sector exportador o líder, además de estimar la peligrosidad de sus competidores.

En su artículo sobre la competitividad, Vial (2002) define dicho concepto como "la capacidad para sostener tasas de crecimiento elevadas por un período de tiempo prolongado". Además, en relación a los componentes que se requieren para generar esta tasa elevada se indican:

- Las condiciones geográficas, que en este estudio están relacionadas con la localización de los países productores de fruta del hemisferio sur. Estas condiciones determinan interacciones estratégicas y determinantes respecto de la variable clima y medio ecológico que requieren los frutales para impulsar fuertes impactos en la productividad de los factores productivos (tierra, trabajo, capital y capacidad organizativa). Las condiciones de localización geográfica de las zonas productoras de fruta de contraestación son una variable a considerar para establecer los envíos de fruta a los mercados del hemisferio norte, donde se localiza la demanda, y que hace que los costos de transporte por vía marítima o aérea sean una restricción para la colocación de la fruta en los puertos de ingreso.

- Integración a la economía mundial. Esta condición adquiere mayor importancia cuando los países definen estrategias de desarrollo orientadas al comercio internacional y sus envíos de fruta se insertan a los flujos mundiales de comercio; la experiencia internacional indica que los países que han tenido éxito se han insertado en las corrientes de comercio del sistema mundial.

- Estabilidad macroeconómica. Los diversos estudios convergen en indicar que las sinergias que se producen con la estabilidad macroeconómica generan confianza en los inversores, en la tasa de ahorro interno, una tasa de inflación baja y un tamaño del gobierno relativamente pequeño, que hace que este conjunto de variables favorezcan el crecimiento.

- Instituciones apropiadas, esto implica que impere el principio de la ley, el resguardo a la propiedad, la ausencia de corrupción, que hacen que las instituciones con su trabajo generen las sinergias para el crecimiento económico.

- Capacidad de innovación y de absorción de tecnología, en la actualidad marcha a gran velocidad, lo que obliga a los países y a las empresas a estar muy atentas a las innovaciones tecnológicas y a absorber las nuevas tecnologías, que se desarrollan en los distintos ámbitos productivos, las cuales están asociadas a generar nuevos productos, a la diferenciación de los productos y a reducir los costos de producción.

En definitiva, el conjunto de estos factores ha permitido construir los índices agregados que acceden para comparar a los países en un momento dado del tiempo; esta información se expresa en el Índice de Competitividad para el Crecimiento (ICC), el cual aún no integra la componente geográfica, pero entrega una identificación de los puntos fuertes y débiles del sistema económico y de las instituciones de los países.

Los tres componentes del Índice de Competitividad para el Crecimiento (ICC): Ambiente Macroeconómico, Calidad de las Instituciones Públicas y Tecnología, junto a sus respectivos subcomponentes y las ponderaciones correspondientes, se presentan en el Cuadro 1, cuyas ponderaciones difieren, cuando se trata de naciones innovadoras (países con más de 15 patentes por millón de habitantes) de las otras naciones no innovadoras, en particular en el subcomponente innovación, que cambia de 0,125 a 0,5 su peso relativo de ponderación. En el caso de los países que se encuentran en estudio, es el componente Tecnología el que refleja mejor la incidencia de este componente en el ICC, por los impactos de la innovación, el uso de tecnologías de información y comunicación y la transferencia tecnológica, que en su conjunto impactan al proceso productivo que se orienta a la exportación.

Además, el informe sobre competitividad del Foro Económico Mundial (2004) enfatiza el rol que juega la tecnología en los procesos de crecimiento en los diferentes países, dependiendo de su particular estado de desarrollo. A saber, está ampliamente aceptado que la innovación tecnológica es relativamente más importante para el crecimiento de los países que están en la frontera del conocimiento. Así, en los países más adelantados es más importante la innovación y en los países de desarrollo intermedio la importancia está dada por la adopción de tecnología extranjera o la transferencia tecnológica asociada a la inversión extranjera directa. 


\section{Cuadro 1}

Componentes del Índice de Competitividad para el Crecimiento, ICC, y sus ponderaciones (Expresado en porcentaje)

\begin{tabular}{|l|l|}
\hline \multicolumn{2}{|c|}{ Índice de Competitividad para el Crecimiento } \\
\hline $\begin{array}{l}\text { Ambiente Macroeco- } \\
\text { nómico }\end{array}$ & Estabilidad macroeconómica: \\
$0,25(0,33)$ & 0,50 \\
& Riesgo país: 0,25 \\
& Gastos improductivos del gobierno: \\
& 0,25 \\
\hline Calidad Instituciones & Corrupción: 0,50 \\
Públicas & Contratos y leyes: 0,50 \\
$0,25(0,33)$ & \\
\hline Tecnología & Innovación: $0,5(0,125)$ \\
$0,50(0,33)$ & TIC: $(0,375)$ \\
& Transferencia Tecnológica: 0,5 \\
& $(0,5)$ \\
\hline
\end{tabular}

El valor indicado entre paréntesis corresponde a países considerados no líderes en innovaciones.

Fuente: Foro Económico Mundial, 2004.
La información sobre el ICC y la posición relativa dentro de los países productores de fruta del hemisferio sur se presenta en relación al decil respectivo, en atención a los cambios que ha incorporado el Foro Económico Mundial en cuanto al número de países analizados y ajustes a la metodología seguida en las ponderaciones en los componentes del ICC.

El Cuadro 2 reporta la ubicación alcanzada por cada uno de los países exportadores de fruta del hemisferio sur en el ranking del índice ICC, donde se compara su posición relativa en los respectivos deciles alcanzados durante el período 2000-2004. Así se comprueba que en el caso de Australia se ha posicionado entre el primer y segundo decil. Mientras que Nueva Zelandia se ha mantenido en el segundo decil desde 2001 hasta 2004; Chile a su vez ha evolucionado desde el cuarto al tercer decil; Brasil no ha cambiado su posición en el sexto decil durante todo el período de análisis; Sudáfrica, sin embargo, se ubica entre el quinto y cuarto decil y finalmente Argentina, que claramente ha perdido posiciones, desde el sexto al octavo decil.

Cuadro 2

Ranking de competitividad para el crecimiento, ICC, de los países exportadores de fruta del hemisferio sur (Expresado en deciles)

\begin{tabular}{|l|c|c|c|c|c|}
\hline \multicolumn{1}{|c|}{ Países } & $\mathbf{2 0 0 0}$ & $\mathbf{2 0 0 1}$ & $\mathbf{2 0 0 2}$ & $\mathbf{2 0 0 3}$ & $\mathbf{2 0 0 4}$ \\
\hline Australia & $2^{\circ} \mathrm{d}$ & $1^{\circ} \mathrm{d}$ & $1^{\circ} \mathrm{d}$ & $1^{\circ} \mathrm{d}$ & $2^{\circ} \mathrm{d}$ \\
Argentina & $6^{\circ} \mathrm{d}$ & $6^{\circ} \mathrm{d}$ & $8^{\circ} \mathrm{d}$ & $8^{\circ} \mathrm{d}$ & $8^{\circ} \mathrm{d}$ \\
Brasil & $6^{\circ} \mathrm{d}$ & $6^{\circ} \mathrm{d}$ & $6^{\circ} \mathrm{d}$ & $6^{\circ} \mathrm{d}$ & $6^{\circ} \mathrm{d}$ \\
Chile & $4^{\circ} \mathrm{d}$ & $4^{\circ} \mathrm{d}$ & $3^{\circ} \mathrm{d}$ & $3^{\circ} \mathrm{d}$ & $3^{\circ} \mathrm{d}$ \\
N. Zelandia & $3^{\circ} \mathrm{d}$ & $2^{\circ} \mathrm{d}$ & $2^{\circ} \mathrm{d}$ & $2^{\circ} \mathrm{d}$ & $2^{\circ} \mathrm{d}$ \\
Sudáfrica & $5^{\circ} \mathrm{d}$ & $5^{\circ} \mathrm{d}$ & $4^{\circ} \mathrm{d}$ & $5^{\circ} \mathrm{d}$ & $5^{\circ} \mathrm{d}$ \\
\hline
\end{tabular}

Fuente: Elaboración propia sobre cifras del Foro Económico Mundial, 2000-2004.

Cuadro 3

Ranking de competitividad para los negocios, ICN, de los países exportadores de fruta del hemisferio sur (Expresado en deciles)

\begin{tabular}{|l|c|c|c|c|}
\hline \multicolumn{1}{|c|}{ Países } & $\mathbf{2 0 0 1}$ & $\mathbf{2 0 0 2}$ & $\mathbf{2 0 0 3}$ & $\mathbf{2 0 0 4}$ \\
\hline Australia & $2^{\circ} \mathrm{d}$ & $2^{\circ} \mathrm{d}$ & $2^{\circ} \mathrm{d}$ & $2^{\circ} \mathrm{d}$ \\
Argentina & $7^{\circ} \mathrm{d}$ & $9^{\circ} \mathrm{d}$ & $6^{\circ} \mathrm{d}$ & $8^{\circ} \mathrm{d}$ \\
Brasil & $4^{\circ} \mathrm{d}$ & $5^{\circ} \mathrm{d}$ & $4^{\circ} \mathrm{d}$ & $4^{\circ} \mathrm{d}$ \\
Chile & $4^{\circ} \mathrm{d}$ & $4^{\circ} \mathrm{d}$ & $4^{\circ} \mathrm{d}$ & $2^{\circ} \mathrm{d}$ \\
N. Zelandia & $3^{\circ} \mathrm{d}$ & $3^{\circ} \mathrm{d}$ & $2^{\circ} \mathrm{d}$ & $3^{\circ} \mathrm{d}$ \\
Sudáfrica & $4^{\circ} \mathrm{d}$ & $4^{\circ} \mathrm{d}$ & $3^{\circ} \mathrm{d}$ & \\
\hline
\end{tabular}

Fuente: Elaboración propia sobre cifras del Foro Económico Mundial, 2001-2004. 
En el Cuadro 3 se presenta la información sobre el ICN de cada uno de los países productores de fruta del hemisferio sur, en relación al decil respectivo. En el caso de Australia, este país ha estado posicionado en el segundo decil durante todo el período de análisis. Mientras que Nueva Zelandia ha evolucionado desde el tercer decil al segundo; Chile a su vez ha avanzado desde el cuarto decil al tercero; Brasil, por su parte, ha cambiado de posición entre el cuarto y quinto decil; Sudáfrica, sin embargo, se ubica entre el cuarto y tercer decil y finalmente Argentina que definitivamente ha perdido posiciones desde el sexto al octavo decil y en el año 2002 alcanza su posición más desfavorable en el noveno decil.

Por otra parte, es importante lo planteado por Ballarin (1997), al relacionar el proceso de globalización con el liderazgo tecnológico en el sector y las cuotas de mercado que los países logran alcanzar, indicando que estas para mantenerlas requieren el respaldo de un vigoroso proceso de investigación.

El Cuadro 4 permite realizar el cruce de información entre la participación porcentual en el mercado de cada especie con los índices ICC y ICN. En consecuencia, en el caso de la uva, Chile es el líder con el 72,1\% del mercado y Sudáfrica prácticamente participa con el $20 \%$ del mercado; respecto del ICC, Chile está en mejor ubicación (22) en relación con Sudáfrica (41), en cuanto al índice ICN, Chile y Sudáfrica están en posiciones muy próximas, 29 y 25, respectivamente. Además, en cuanto a esta especie, Sudáfrica es el país que amenaza la posición del líder, pero tan solo con un $20 \%$ de participación del mercado y dadas las posiciones relativas de los índices ICC y ICN la situación marca una tendencia de estabilidad entre estos dos países competidores. Más aún, al considerar los índices ICC y ICN, Australia es el país de mejores expectativas para amenazar a los principales líderes, pero tan solo tiene un 3,5\% de la participación del mercado. También, Argentina por la ubicación en los índices ICC y ICN (74), ubicación que está reflejando el difícil momento económico que atraviesa, se estima no se constituiría en una amenaza para el líder del mercado en los próximos años.

De igual forma, en cuanto al mercado de las peras, es una situación interesante, ya que el líder, Argentina, con una participación del 47,7\% del mercado exportador del hemisferio sur, se encuentra afectado por una fuerte crisis económica. Todavía más, en el ICC y en el ICN se ubica en el lugar 74 entre 104 países estudiados. Le siguen Chile (ICC, 22 y ICN, 29) y Sudáfrica (ICC, 41, ICN, 25; ambos países muestran sus condiciones competitivas para generar las condiciones necesarias para amenazar al líder, que podrá continuar en una condición inercial por los próximos años, aprovechando la evolución de las plantaciones ya existentes. En conclusión, en el mercado de peras del hemisferio sur se presentan expectativas de reducir la participación del líder por parte de sus competidores más cercanos.

Por lo demás, en el caso del mercado de las manzanas, Chile es el líder con el 33,2\% de participación en el mercado, luego Nueva Zelandia que participa con el 29,9\% y Sudáfrica que participa del 20,1\%; respecto del ICC, Nueva Zelandia está en mejor ubicación (ICC, 18 y ICN, 18) contra Chile (ICC, 22 y ICN, 29) y Sudáfrica (ICC, 41 y ICN, 25). En suma, en cuanto a esta especie, Nueva Zelandia y Sudáfrica son los países que

\section{Cuadro 4}

Participación porcentual por especie en el mercado exportador del hemisferio sur y los Índices ICC, ICN, 2004

\begin{tabular}{|l|c|c|c|c|c|c|c|}
\hline \multicolumn{1}{|c|}{ Países } & Uva & Peras & Manz. & Palta & Kiwi & ICC & ICN \\
\hline Argentina & 2,9 & 47,7 & 7,7 & 0 & 0 & 74 & 74 \\
Australia & 3,5 & 3,5 & 2,9 & 0,2 & 0,8 & 14 & 13 \\
Brasil & 1,5 & 0 & 5,2 & 0,4 & 0 & 57 & 38 \\
N. Zelandia & 0 & 2,1 & 29,9 & 5,7 & 68,2 & 18 & 18 \\
Chile & 72,1 & 23,1 & 33,2 & 53,2 & 30,8 & 22 & 29 \\
Sudáfrica & 19,6 & 18,4 & 20,1 & 32,2 & 0 & 41 & 25 \\
\hline
\end{tabular}

Fuente: Foro Económico Mundial, para los índices ICC y ICN; ODEPA para los antecedentes de producción de Chile y FAO para los otros países, 2004. 
amenazan la posición del líder. Así, por ejemplo, el mercado de la manzana marca una tendencia de alta competencia entre estos tres competidores. $\mathrm{Al}$ considerar los índices ICC y ICN, es Nueva Zelandia el país con mejores expectativas para amenazar y alcanzar al líder.

De igual forma, el mercado de la palta es dominado en un 53,2\% por Chile, seguido por Sudáfrica con el 32,2\%. Al considerar los índices ICC, Chile supera a Sudáfrica, 22 y 41 respectivamente, pero en el índice ICN Sudáfrica supera a Chile 25 y 29, lo que refleja que Sudáfrica tiene leves mejores condiciones para organizar los negocios. En suma, en función de estos antecedentes, este mercado de la palta mantendrá la evolución que ha venido mostrando en los últimos años, donde Nueva Zelandia debería crecer en su participación, que alcanza hoy tan solo un $5,7 \%$, dados sus mejores indicadores ICC y ICN.

En concreto, el mercado del kiwi es dominado en un $68,2 \%$ por Nueva Zelandia, país que fue el que desarrolló e introdujo este frutal en el mercado mundial, y es seguido por Chile con el 30,8\%. Más aún, al considerar el índice ICC, Nueva Zelandia supera a Chile, 18 y 22 respectivamente, pero en el índice ICN Nueva Zelandia supera a Chile 18 y
29, lo que refleja que Nueva Zelandia tiene mejores condiciones para organizar los negocios, todavía más, la inversión en desarrollo tecnológico en torno al kiwi es importante al establecer una variedad denominada Dorada, que se ha implementado en un esquema de integración vertical, que se constituye desde la fase de propagación hasta la comercialización en el mercado internacional, con una organización en la comercialización que expresa la identidad nacional (Board), World Kiwifruit Review (2004). En conclusión, en función de estos antecedentes este mercado del kiwi mostrará una tendencia a consolidar y ampliar la trayectoria que ha venido mostrando Nueva Zelandia en los últimos años; además Australia debería crecer en su participación, que hoy alcanza tan solo un $0,8 \%$.

En la Figura 1 se reporta la participación porcentual del país líder en el mercado de cada especie frutal, destacando con alrededor del $70 \%$, en sus respectivas especies, Chile en uva y Nueva Zelandia en kiwi. Además Chile es el líder en el mercado de paltas, superando levemente el 50\%, y mantiene el liderazgo en manzanas con el 33\% de participación del mercado. Argentina es el líder en el mercado de las peras con el $47 \%$ de participación en el mercado.

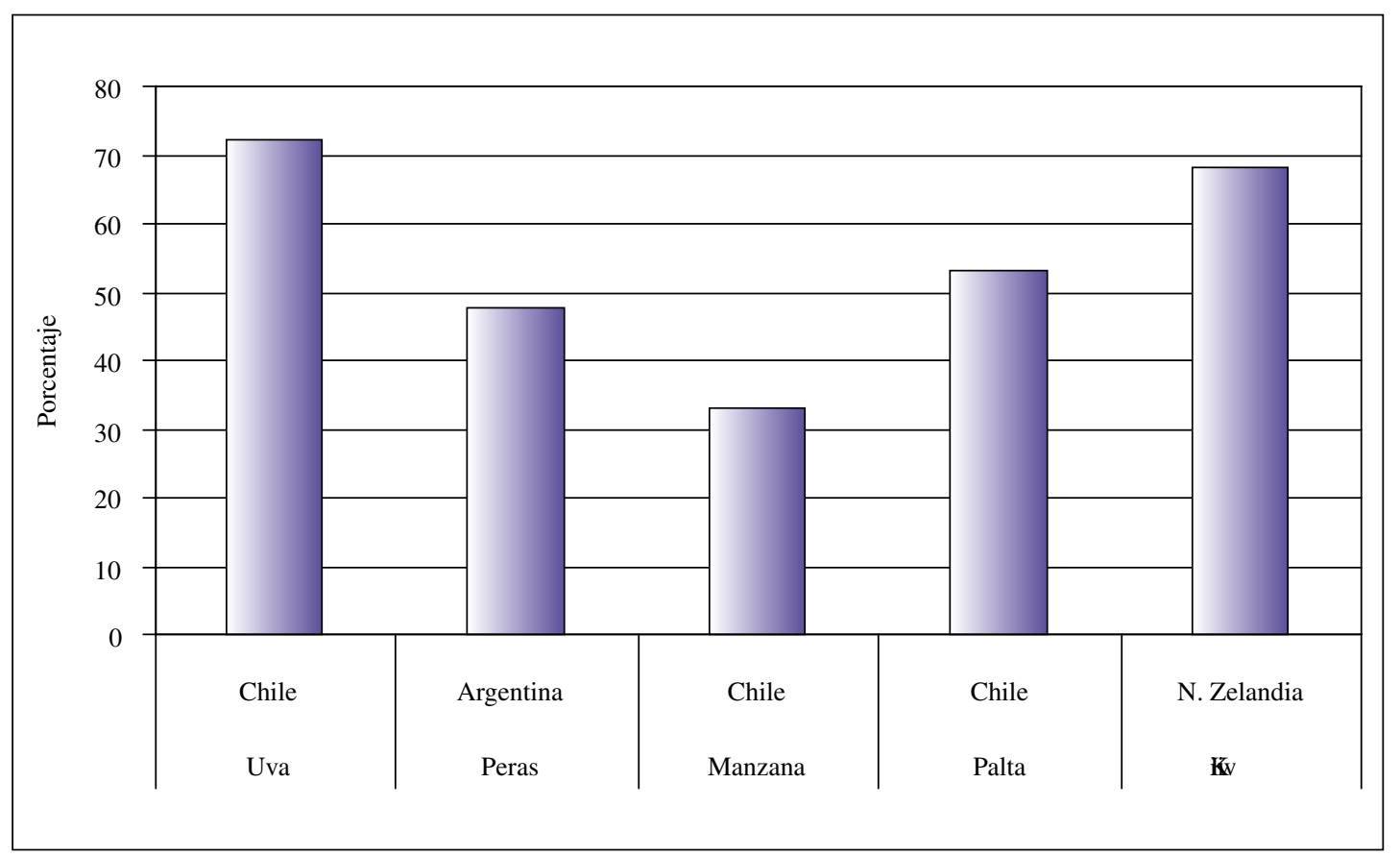

Figura 1. Participación porcentual del país líder en el mercado de cada especie frutal, 2003 (Expresado en porcentaje). Fuente: Elaboración propia a partir de los datos recopilados para 2003. 
El análisis de los datos obtenidos sobre la participación porcentual en el mercado de los países que compiten en el hemisferio sur en cada especie frutal y su relación con el índice ICC. permite diferenciar cuatro zonas muy características, presentadas en la Figura 2: Zona I, con ICC excelente y participación de mercado baja; Zona II, con ICC deficiente y participación de mercado baja; Zona III, con ICC excelente y participación de mercado alta, y Zona IV, con ICC deficiente y participación de mercado alta, (Tapia, 2006).

Esta situación implica que la ruta crítica hacia la zona 3 determina un tipo de camino específico:

- Desde la zona I, para avanzar a la zona III, se requiere principalmente aumentar la participación de mercado, dado que se cuenta con un buen posicionamiento en el ranking del ICC, con menores ajustes tecnológicos.

- Desde la zona II, para avanzar a la zona III, se requiere avanzar simultáneamente en aumentar la participación de mercado y mejorar el posicionamiento en el ranking del ICC.

- Desde la zona IV, para avanzar a la zona III, se requiere avanzar en mejorar el posicionamiento en el ranking del ICC y no perder la participación de mercado que se posee en determinado rubro.

En definitiva, el tema central pasa por el impacto del componente tecnológico que está definido en el ICC y que en el caso de los países en vías de desarrollo, como Chile, los complica para alcanzar un mejor posicionamiento en el ranking mundial.

\section{MATERIAL Y MÉTODO}

Este es un estudio de tipo descriptivo, que tiene por objetivo indagar sobre la competitividad de los países exportadores de fruta del hemisferio sur a partir de la información que entrega el Índice de Competitividad para el Crecimiento que calcula el Foro Económico Mundial. Esta información se cruza con la participación de mercado para cada especie frutal determinando el liderazgo de un país que domina el mercado de las exportaciones de fruta del hemisferio sur (Hernández, et al. 1998).

Asimismo es una investigación de tipo no experimental, puesto que se realiza sin hacer variar intencionalmente las variables, se observan los cambios y efectos que sucedieron. Además, es un diseño no experimental longitudinal de tendencia, ya que se desea analizar los cambios a través del tiempo, en cuanto a la conducta de los países y las relaciones entre estos para hacer inferencias respecto al cambio, sus determinantes y consecuencias en distintos momentos en el tiempo. Más aún, el diseño de la investigación es de tipo documental, con medición de datos a partir de información secundaria obtenida de las bases de datos.

De esta forma, se procedió a la recopilación de la información para establecer las series entre 1994-2004 para el índice de competitividad y los valores sobre las exportaciones de las cinco especies más importantes de los envíos de Chile. Estos datos sobre exportaciones se recopilan a partir de la base de datos del Ministerio de Agricultura (ODEPA, 1993-2004); las exportaciones de los otros países del hemisferio sur se obtuvieron de la base de datos de FAO. La información obtenida se tabula en gráficos a fin de determinar la ubicación del país

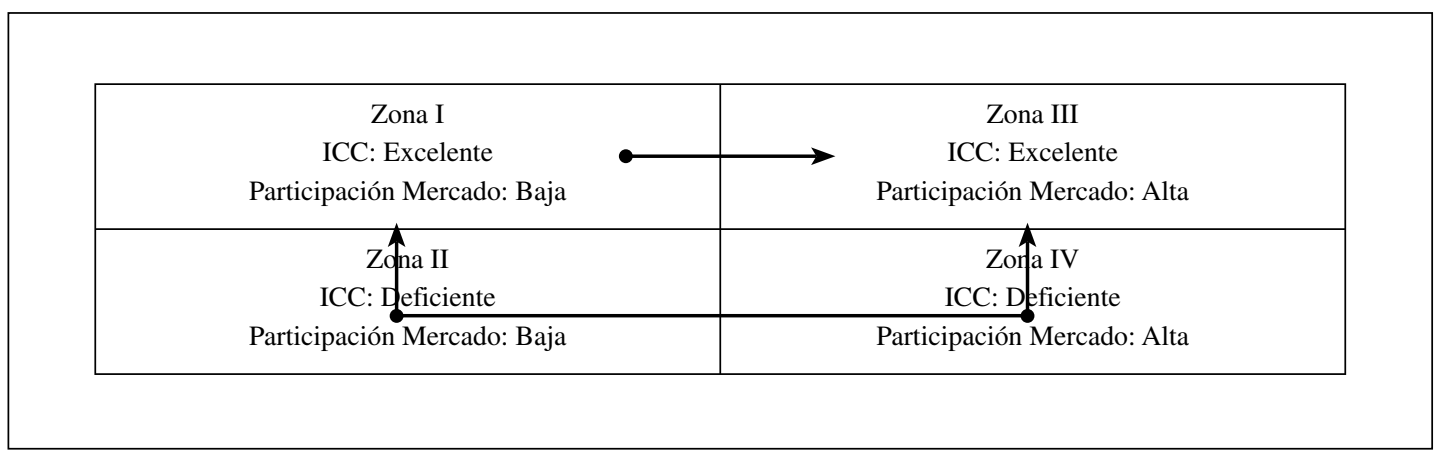

Figura 2. Determinación de zonas características en la relación entre participación de mercado y ranking de posición en el ICC. Fuente: Elaboración propia. 
en los cuadrantes característicos en función del ICC y la participación de mercado para las frutas en estudio.

\section{RESULTADOS}

La Figura 3 muestra la evolución en el ranking de deciles de los países exportadores de fruta del hemisferio sur en el ICC. Es importante indicar que al año 2004 tanto Australia como Nueva Zelandia se encuentran en el primer decil; Chile, por su parte, avanza desde el cuarto al tercer decil, Sudáfrica, a su vez, se mantiene en el cuarto, Brasil permanece en el sexto decil y finalmente Argentina muestra inestabilidad después de su caída del año 2002, permaneciendo en el octavo decil.

La figura 4 muestra la evolución en el ranking de deciles de los países exportadores de fruta del hemisferio sur en el ICN. Es importante indicar que al año 2004 tanto Australia como Nueva Zelandia se encuentran en el segundo decil; Chile, por su parte, avanza al tercer decil, compartiendo esta posición con Sudáfrica. Brasil, a su vez, permanece en el cuarto decil y finalmente Argentina muestra inestabilidad cayendo desde el sexto al octavo decil.

Al considerar el componente tecnológico del Índice de Competitividad para el crecimiento, ICC, se puede inferir que tanto Australia como Nueva Zelandia son los países que tienen la mayor capacidad para lograr un crecimiento sostenido de sus economías en el mediano plazo y por lo tanto de su sector frutícola, dadas las condiciones alcanzadas en su ambiente macroeconómico, en sus instituciones públicas y la tecnología que estos países disponen, lo que permite estimar el potencial de competitividad de cada país en el mercado frutícola exportador del hemisferio sur.

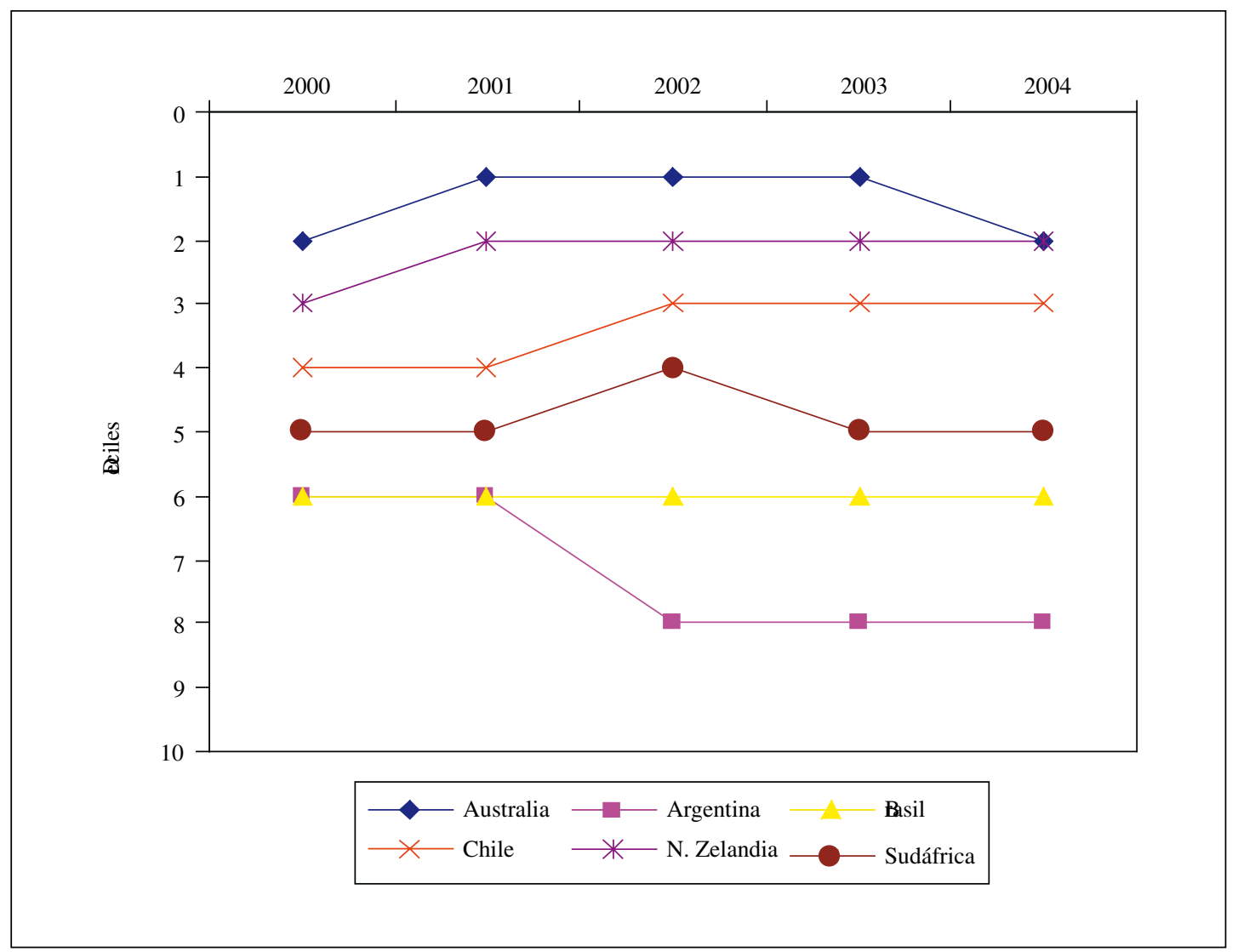

Figura 3. Ranking de competitividad para el crecimiento, ICC, para los países exportadores de fruta del hemisferio sur en la ubicación en deciles, 2000-2004 (Expresado en deciles).

Fuente: Elaboración propia sobre cifras del Foro Económico Mundial, 2000-2004. 


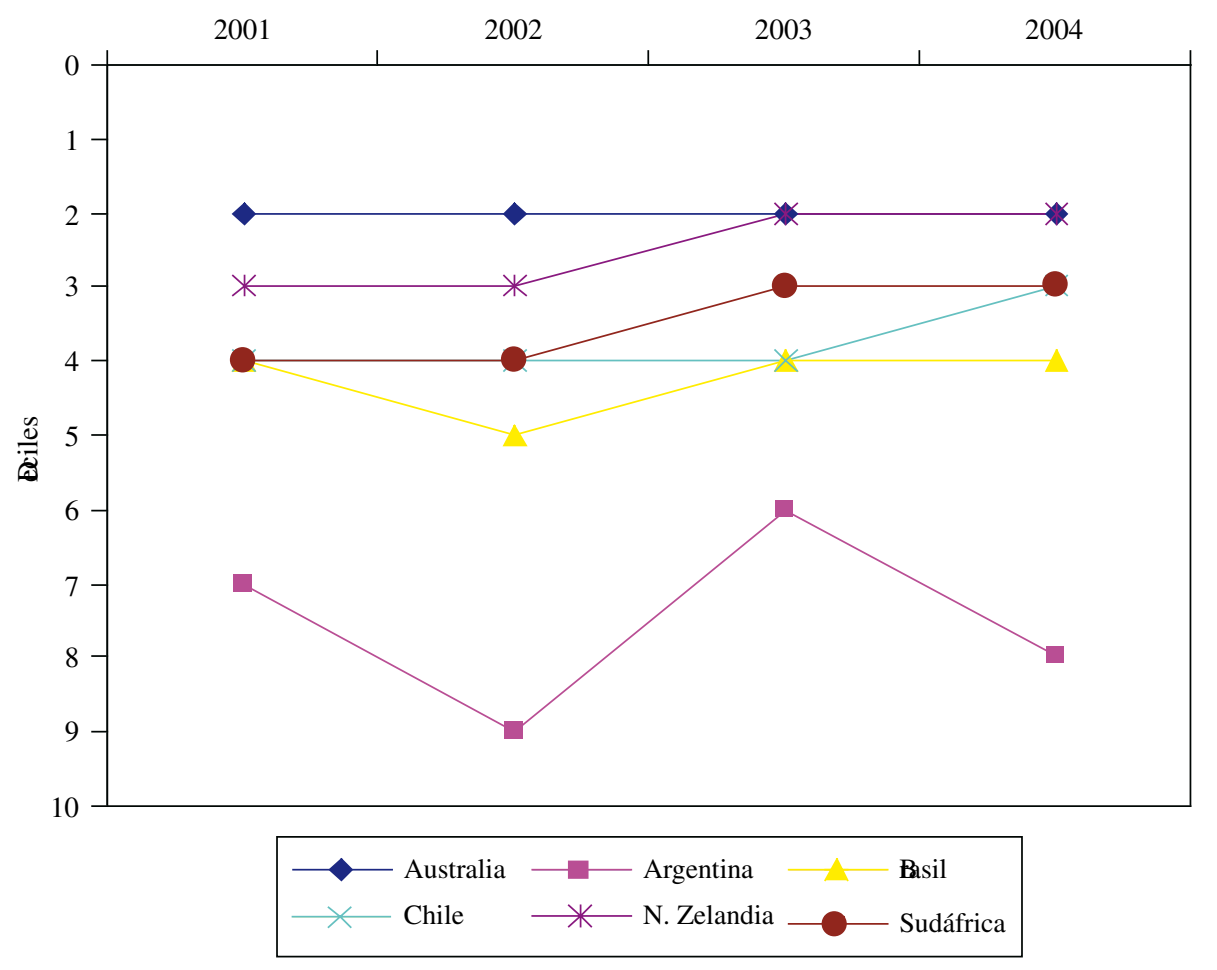

Figura 4. Ranking de competitividad para el crecimiento de los negocios, ICN, para los países exportadores de fruta del hemisferio Sur en la ubicación en deciles (Expresado en deciles).

Fuente: Elaboración propia sobre cifras del Foro Económico Mundial, 2001-2004.

En la figura 5 se presenta la situación del componente tecnológico para el año 2004 para los países exportadores de fruta del hemisferio sur donde Australia (17) y Nueva Zelandia (24) son los países mejor ubicados, les siguen Chile (32), Sudáfrica (40) y finalmente Argentina, en el lugar 57 dentro de 104 países analizados.

En consecuencia, tanto Australia como Nueva Zelandia son países capaces de generar tecnología en procesos innovadores, especialmente en el sector agrícola, en tanto Chile, Argentina y Brasil son países que crecen adaptando tecnología desarrollada en el extranjero.

En resumen, el componente tecnológico del índice ICC muestra un mejor resultado para Australia y Nueva Zelandia, comparado con Chile, y éste, a su vez, supera a Brasil, Sudáfrica y Argentina. Este último país es el que muestra los mayores retrocesos en este período. Otra metodología sobre este tema son los criterios que utiliza el Banco Mundial para evaluar a los países utilizando una amplia batería de indicadores para estimar la situación científicotecnológica de los países (Benavente, 2004).

En la Figura 6 se presenta para el caso de Chile, en el esquema de las zonas descritas anteriormente, la ubicación de las XF5. En la zona 3 solamente se encuentran la uva y palta, mientras que manzana, kiwi y peras están en la zona 1 y la ruta crítica para esta tres especies indica que se requiere avanzar en los aspectos tecnológicos de estas especies y simultáneamente alcanzar un mejor posicionamiento en la participación de mercado.

La Figura 7 presenta los resultados de la participación de mercado de las XF5 para los países exportadores de fruta del hemisferio sur, incluyendo a los tres principales países competidores, relacionando esta información con los valores que alcanza el ranking en el índice ICC y la participación de 


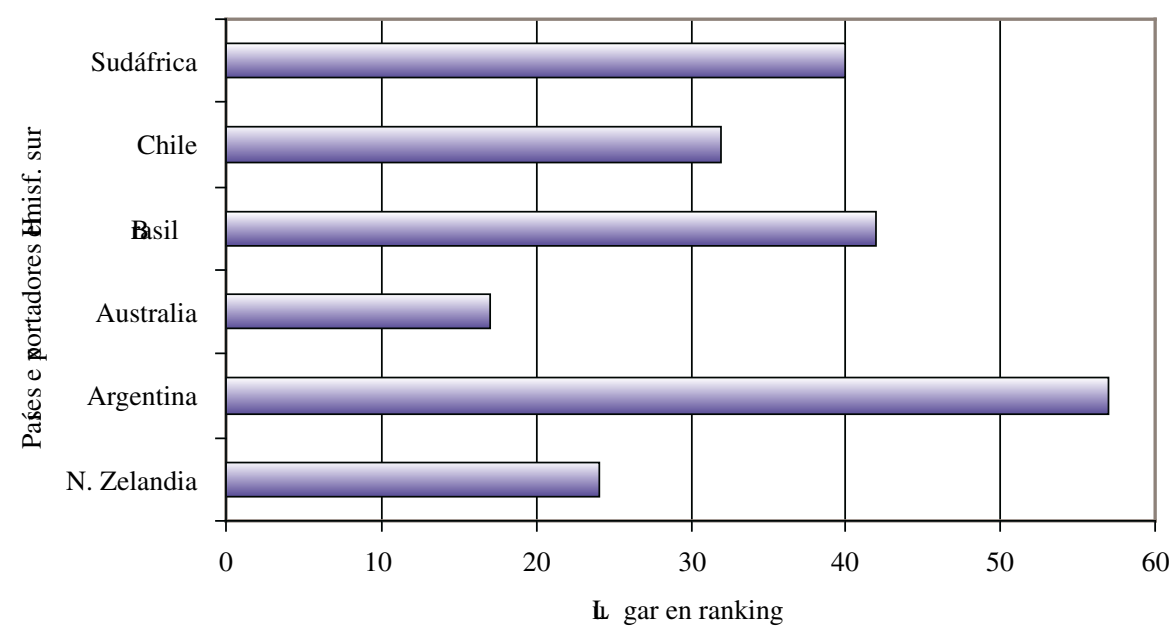

Figura 5. Ranking de competitividad para el crecimiento, ICC, componente tecnológico para los países exportadores de fruta del hemisferio sur, 2004 (Expresado en lugar en ranking).

Fuente: Elaboración propia sobre cifras del Foro Económico Mundial, año 2004.

Porcentaj de participación de mercado

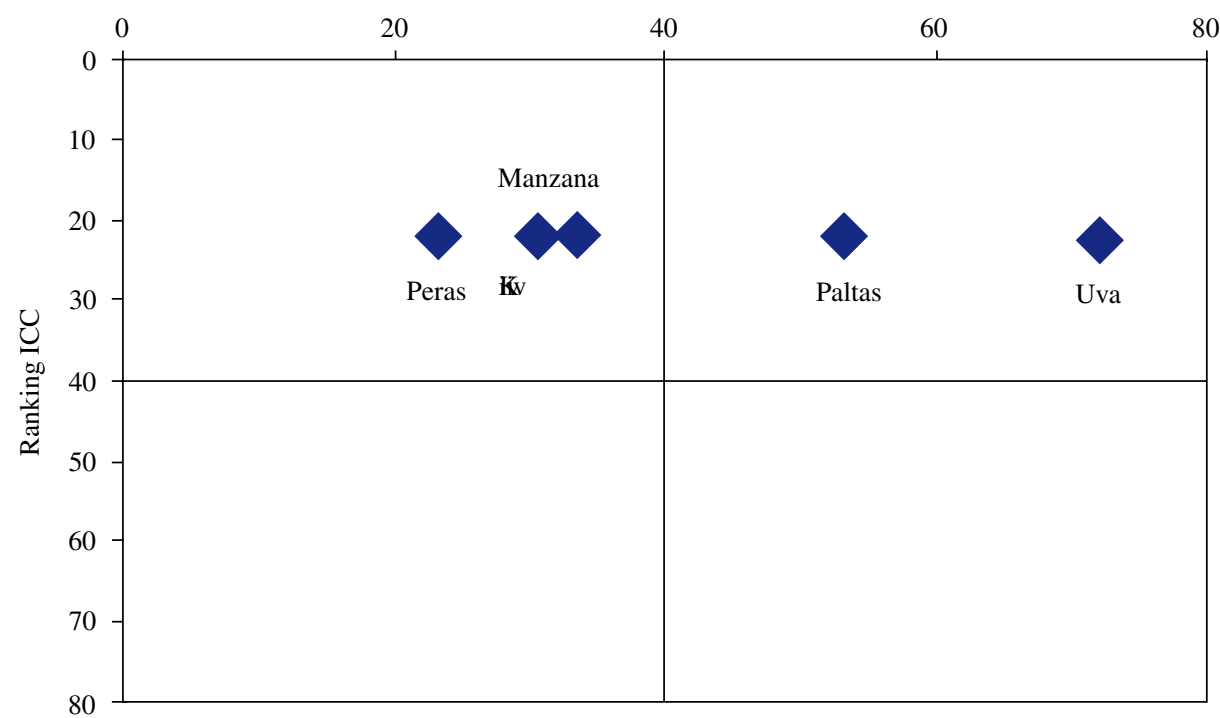

Figura 6. Determinación de zonas características en la relación entre la participación de mercado y ranking de posición en el ICC para Chile en las XF5.

Fuente: Elaboración propia a partir de los datos obtenidos. 

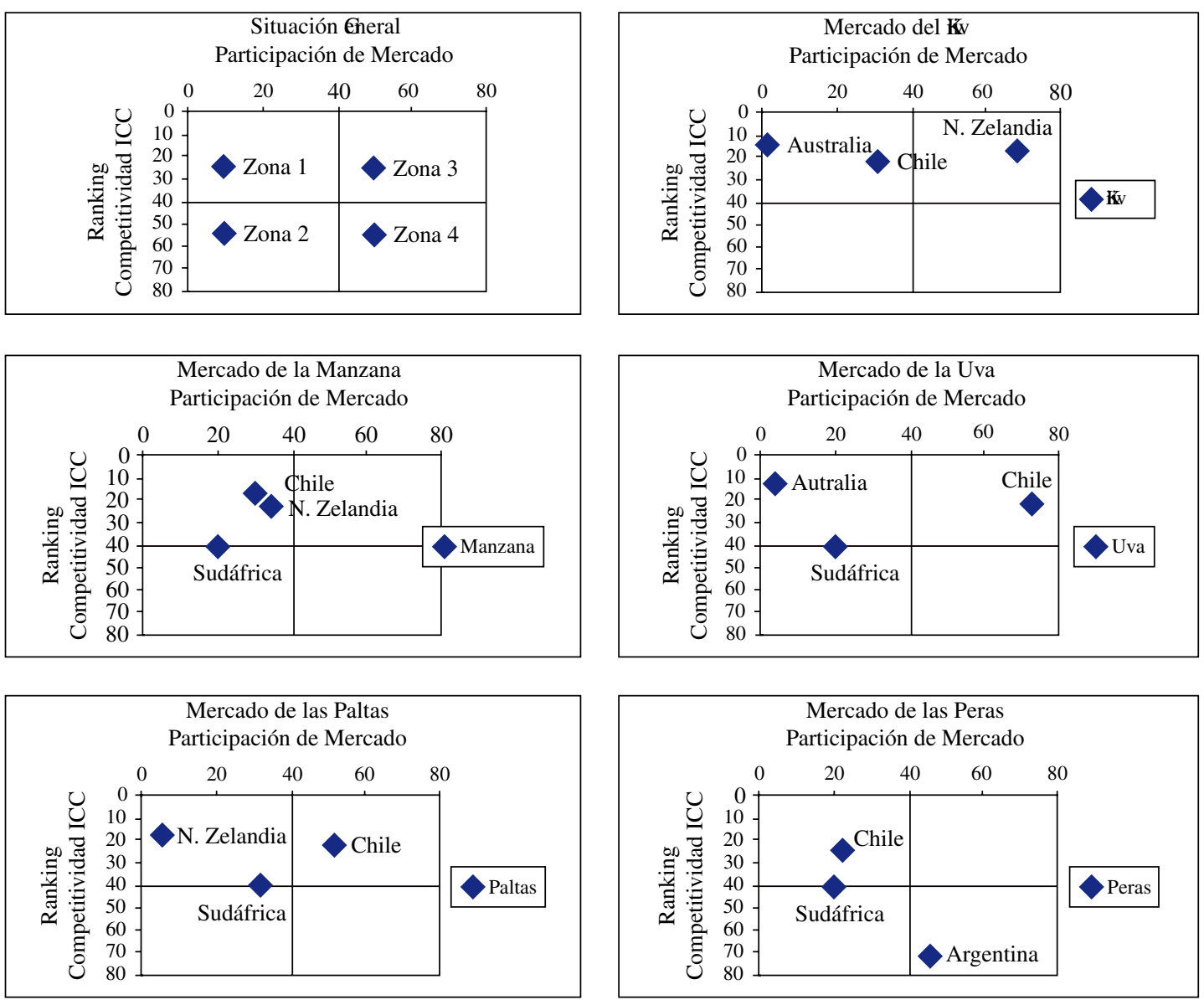

Figura 7. Participación porcentual en el mercado de los países competidores en las XF5 y su relación con el índice ICC en las zonas analizadas.

Fuente: Elaboración propia a partir de datos del Foro Económico Mundial, ODEPA y FAO.

mercado para cada país. Así, se pueden diferenciar el cuadrante donde se encuentra el líder y sus competidores.

En el caso del mercado del kiwi, tan solo Nueva Zelandia se ubica en el tercer cuadrante, Chile, su competidor más cercano, aún se encuentra en el cuadrante 1. El mercado de la manzana es el más competitivo de todos, no hay países en el tercer cuadrante. El mercado de la uva ubica tan solo a Chile en el tercer cuadrante, mientras que Sudáfrica está aún en el límite del segundo cuadrante. La situación del mercado de las paltas mantiene a Chile en el tercer cuadrante, mientras que Sudáfrica se encuentra en el límite del segundo cuadrante. Finalmente en el mercado de las peras no hay países en el tercer cuadrante y tanto Chile como Sudáfrica están amenazando al líder que está en el cuarto cuadrante.

\section{CONCLUSIONES}

Este estudio permite concluir que a partir de la información que se genera en el posicionamiento mundial del ICC y la participación de mercado del hemisferio sur, para cada una de las XF5, se puede estimar la ubicación del país líder y sus más cercanos competidores en alguno de los cuatro cuadrantes. La situación ideal de ubicación en el tercer cuadrante solo es posible observarla en el mercado del kiwi, uva y palta con su respectivo país líder.

Otra conclusión de este estudio indica que Argentina es el país exportador de fruta del hemisferio sur que muestra más retrocesos en este período y su condición de líder en el mercado de las peras podrá continuar en una condición inercial por los próximos años, aprovechando la evolución de las plantaciones ya existentes. Por lo tanto, en 
el mercado de peras del hemisferio sur hay expectativas de reducir la participación del líder de sus competidores más cercanos.

Asimismo, el mercado de la uva es dominado por Chile y el del kiwi por Nueva Zelandia, situación que corresponde a una interesante combinación entre una alta participación en el mercado y un posicionamiento en el ranking mundial del ICC menor de 20.

En cuanto al mercado de la manzana, se produce una situación de participación de mercado relativamente equivalente para los tres países, Chile, Nueva Zelandia y Sudáfrica, y al considerar el ICC, Nueva Zelandia está mejor posicionado que Chile y Sudáfrica, lo que le permitiría posicionarse mejor en este mercado a futuro.

Además, al considerar el ICC en los distintos años, se infiere que tanto Australia como Nueva Zelandia son los países que tienen la mayor capacidad para lograr un crecimiento sostenido de sus economías en el mediano plazo y por lo tanto de su

\section{LITERATURA CITADA}

BALLARIN, E. (1997). “Globalización frente a Localización: Un dilema estratégico”. IESE. Universidad de Navarra, España.

BENAVENTE, H.; J. M. (2004). "Innovación Tecnológica en Chile. Donde estamos y qué se puede hacer", Documento de Trabajo No 295, Banco Central de Chile, Santiago, Chile.

FORO ECONÓMICO MUNDIAL. Índice de Competitividad Global, Índice de Competitividad para el Crecimiento (GCI), Índice de Competitividad para los Negocios (BCI), para los diferentes países del mundo, años 2000, 2001, 2003 y 2004.

HERNÁNDEZ R.; FERNÁNDEZ C.; BAPTISTA P. (1998) Metodología de la Investigación, Editorial Mc Graw-Hill, México.

OFICINA DE ESTUDIOS Y POLÍTICAS AGRARIAS, ODEPA 1993-2004. Base de datos de exportaciones agropecuarias de Chile, Santiago, Chile, disponible en www.odepa.cl sector frutícola, dadas las condiciones alcanzadas en sus ambientes macroeconómicos, sus instituciones públicas y la tecnología que generan.

En consecuencia, tanto Australia como Nueva Zelandia son países que son capaces de generar tecnología en procesos innovadores, especialmente en el sector agrícola, en tanto Chile, Argentina y Brasil son países que crecen adaptando tecnología desarrollada en el extranjero.

El componente tecnológico del ICC es la variable que muestra mejor la condición para desarrollar conocimiento innovador y aplicarlo en el sector y obtener ventajas competitivas por esta vía, el ejemplo más claro es Nueva Zelandia en kiwi.

Finalmente, los resultados obtenidos permiten establecer una ruta crítica para elegir algunas de las estrategias para alcanzar un mejor posicionamiento para el país y el frutal respectivo, tratando de alcanzar el tercer cuadrante, donde se obtiene una alta participación de mercado y un posicionamiento en el ranking mundial del ICC menor de 20.

\section{ORGANIZACIÓN MUNDIAL PARA LA ALIMENTACIÓN} Y LA AGRICULTURA, FAO. Base de datos sobre exportaciones agrícolas de los países. Disponible en: http://www. fao.org/faostat

PORTER, M. (1991). La ventaja competitiva de las naciones, Editor Javier Vergara S.A., Buenos Aires, Argentina.

TAPIA, I. L. (2006). Evaluación de las condiciones de competitividad del sector frutícola chileno con relación a su inserción al mercado internacional entre los años 1993-2004. Ediciones Universidad de Tarapacá (en prensa).

VIAL, J. (2002). "Cuán Competitiva es la República Dominicana”. 5 septiembre 2005, <http://portal.onu.org.do/contenidos/archivos/Cuan\%20competitiva\%20es\%20RD.pdf>

WORLD KIWIFRUIT REVIEW (2004). Belrose Publication. USA. 